\title{
Estimating Data-Dependent Jitter of a General LTI System from Step Response
}

\author{
Behnam Analui, James Buckwalter, and Ali Hajimiri \\ California Institute of Technology, Pasadena, CA 91125
}

\begin{abstract}
We present a method for estimating data dependent jitter (DDJ) introduced by a general LTI system, based on the system's step response. A perturbation technique is used to generalize the analytical expression for DDJ. Different scales of DDJ are defined that characterize the probability distribution of jitter. In particular, we identify a dominant prior bit that signifies the well-known distribution of DDJ, the two impulse functions. We also highlight that system bandwidth is not a complete measure for predicting DDJ. We verify our generalized analytical expression of DDJ experimentally and show that estimation errors are less than $7.5 \%$.
\end{abstract}

Index Terms - Timing jitter, data dependent jitter, linear timeinvariant systems, intersymbol interference, transient response.

\section{INTRODUCTION}

Broadband communication at gigahertz speeds relies on understanding of timing jitter. Timing jitter of data transitions are deviations of the threshold-crossing time, i.e. the time at which data crosses a decision threshold, compared to a reference clock. Tails of data jitter distribution cause bit errors even if data is sampled with a jitter-free clock. In addition, the timing jitter of the data is inherited as phase uncertainty of the recovered sampling clock and thus further degrades the bit error rate (BER) of the regenerated data. Bandwidth limitation and dispersion in the transmitter, the channel, and the receiver cause intersymbol interference (ISI) and introduce data dependant jitter (DDJ) to the data timing. DDJ changes the probability distribution function (PDF) of the data jitter and exacerbates link BER, as shown in Fig. 1.

The channel physical medium depends on the application and is typically determined by data rate and communication range. For instance, IEEE 802.3 10GEthernet standard family for local area network [1] proposes using multi-mode fiber, single-mode fiber, or copper transmission line depending on the range. Relating channel characteristics to jitter impact in a general framework provides an efficient design tool for minimizing jitter in these systems.

This work proposes a time domain estimation method for data dependent jitter based on the step response. It can be applied to any channel response to predict the amount of DDJ introduced by the channel. Furthermore, the method can be used to calculate the DDJ impact of various blocks in the transmitter or receiver. The dependence of DDJ on system parameters provides additional insights for designing link blocks that minimize jitter. It also highlights that increasing the bandwidth of a block does not necessarily minimize DDJ. As another benefit, jitter distributions that have been mainly modeled based on histogram measurements [2][3] can be studied analytically.

Our approach is similar to the analysis introduced in [4][5]. We propose an analytical estimation technique for DDJ contri-

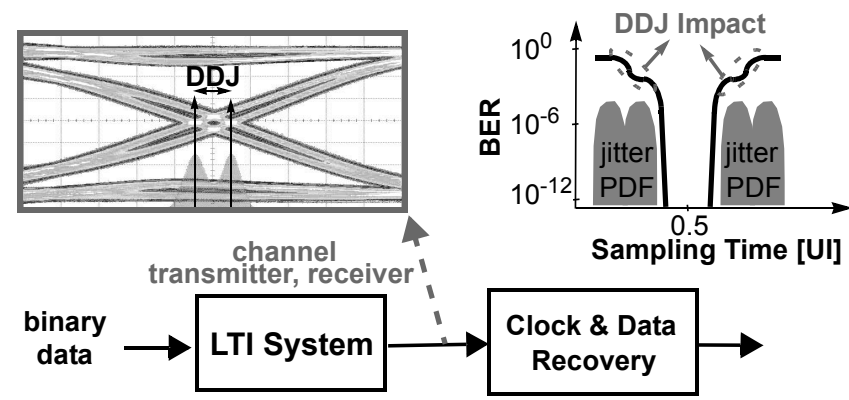

Fig. 1 The block diagram of a communication link with data dependent jitter distribution and its impact on BER

butions of any LTI system and provide experimental verification for our analytical results. We show accurate estimation of DDJ for various transmission lines and circuit blocks based on their step response.

\section{DATA DEPENDENT JITTER}

Data dependent jitter is the deviation of data threshold-crossing time from a reference time due to the residual memory of data bits. Limited bandwidth in the receiver front-end, or channel dispersion, e.g., in FR4 copper transmission line or multimode fiber, cause prior symbols to interfere with the current transition and add ISI and DDJ to the data. Fig. 1 shows the output eye diagram of a microstrip transmission line around the threshold crossing area. The jitter histogram is measured and shows existence of DDJ in both rising and falling edges of the data. We analyze and predict such behavior in this work. In a linear system the rise and fall time transitions are symmetric and analyzing one is sufficient.

The output of a causal LTI system to a random data sequence with bit period $T_{b}$ and a rising transition at $t=0$ can be represented by

$$
y(t)=s(t)+\sum_{k=-\infty}^{-2} a_{k} \cdot p_{o}\left(t-k T_{b}\right),
$$

where $s(t)$ is the step response and models a rising edge when all the prior bits are zero. $p_{o}(t)$ is the pulse response. The $a_{k} s$ are " 1 " or " $O$ " with a given probability. The sum starts from $k=-2$ and $a_{-1}=0$ to guarantee a rising edge. The solution to

$$
y\left(t_{c}\right)=v_{t h}
$$

for $t_{c}$ is the time of the threshold-crossing event as a function of data statistics and system parameters. We compare $t_{c}$ to the 
time of threshold-crossing event when all the $a_{k} s$ are zero and denote it by $t_{0}$. DDJ is defined as

$$
\Delta t \equiv t_{0}-t_{c} .
$$

The analysis of the solution to (2) for a first order LTI system is insightful. From [4] and assuming $v_{t h}=0.5$ we have

$$
\Delta t=-\tau \cdot \ln \left(1-\left(\frac{1-\alpha}{\alpha}\right) \sum_{k=-\infty}^{-2} a_{k} \cdot \alpha^{-k}\right)
$$

where $\tau$ is the system time constant and $\alpha \equiv e^{-T_{b} / \tau}$ that relates the system bandwidth and the bit rate. Equation (4) relates the impact of each prior bit and DDJ. Any prior sequence corresponds to a $\Delta t$ and the overall set of random sequences result in an ensemble of $\Delta t$ values. From (4), the impact of $a_{k}$ on $\Delta t$ vanishes exponentially in a first order system. If only the impact of $a_{-2}$ is included in the calculations, $\Delta t$ can take two sets of values depending on when $a_{-2}$ is " 1 " or " 0 ". Consequently, the data jitter is divided into two mean probability masses, modeled by the two impulse functions [2][4]. The characterizing parameter for this distribution is the distance between the two impulse functions and is called scale-one DDJ, DDJ ${ }_{1}$. Reference [4] calculates $\mathrm{DDJ}_{1}$ for a first order system and demonstrates it excellent agreement with measurement.

Similar behavior for data dependent jitter distribution is generalized to higher order systems as will be seen in section III. A dominant prior bit (not necessarily $a_{-2}$ ) will be identified that shapes data dependent jitter distribution as two impulse functions, as in Fig. 1.

\section{ANALYTICAL EXPRESSION FOR DDJ}

Equation (2) may not be solvable analytically for a general LTI system. We use a technique that approximates DDJ for a general LTI system based only on its step response. Data dependent jitter occurs because the tails of prior bits perturb the time that the data transition crosses the threshold level. If $a_{k}$ is " 1 " the $k$ th prior bit changes $s\left(t_{0}\right)$ by $p_{o}\left(t_{0}-k T_{b}\right)$, in (1). The perturbation shifts the threshold crossing time from $t_{0}$ and causes jitter. Assuming $p_{o}\left(t_{0}-k T_{b}\right) \ll s\left(t_{0}\right)$, the shift in threshold crossing time from the contribution of the $k$ th bit can be calculated from the slope of $s(t)$ at $t_{0}$ and the shift in the amplitude of $s(t)$. This process is shown graphically in Fig. 2 . The time shift due to the $k$ th bit is denoted by $\Delta t_{k}$. We have

$$
\Delta t_{k} \cong-\frac{p_{o}\left(t_{0}-k T_{b}\right)}{\left.\frac{d s(t)}{d t}\right|_{t=t_{0}}}
$$

and the overall perturbation effect, DDJ, is defined as

$$
\Delta t \equiv \sum_{k=-\infty}^{-2} a_{k} \Delta t_{k}=\frac{-1}{\left.\frac{d s(t)}{d t}\right|_{t=t_{0}}} \cdot \sum_{k=-\infty}^{-2} a_{k} p_{o}\left(t_{0}-k T_{b}\right) .
$$

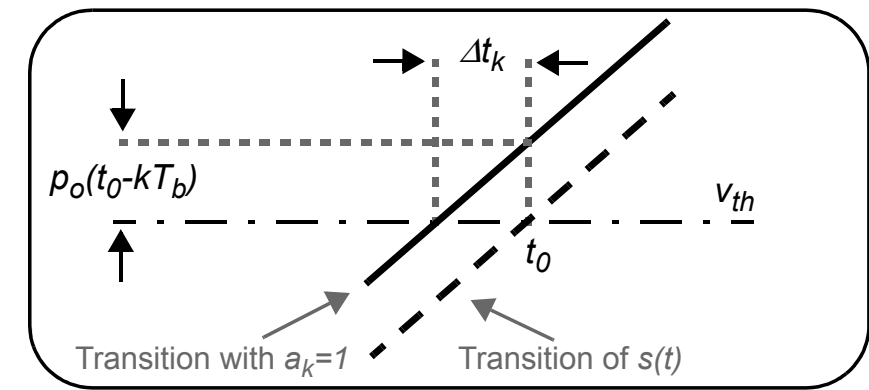

Fig. 2 Deviation of the threshold-crossing time due to the effect of the $k$ th bit

This technique is based on classical perturbation theory. The accuracy of the method is bounded by the perturbation magnitude. In a practical system the bandwidth is chosen such that unit pulse response fall time is within $T_{b}$. Therefore, $p_{o}\left(t_{0}-k T_{b}\right)$ is much smaller than $v_{t h}$ and (5) is a reasonable approximation. We compared this method against the exact result in (4) for a first order system. At the nominal bandwidth to bit rate ratio of 0.7 , the error is only $0.01 \%$. The worst case error is $2.5 \%$.

We can use (6) to estimate the peak-to-peak DDJ and DDJ 1 of a general LTI system. We have

$$
\Delta t_{p p}=\max \{\Delta t\}-\min \{\Delta t\}
$$

The maximum and minimum of $\Delta t$ are achieved for the data sequences in which $a_{k}=1$ only if $p_{o}\left(t_{0}-k T_{b}\right) \leq 0$ and $p_{o}\left(t_{0}-k T_{b}\right) \geq 0$, respectively. Therefore, (7) is simplified to

$$
\Delta t_{p p}=\frac{1}{\left.\frac{d s(t)}{d t}\right|_{t=t_{0}}} \cdot \sum_{k=-\infty}^{-2}\left|p_{o}\left(t_{0}-k T_{b}\right)\right|
$$

Scale-one DDJ can also be defined for a general LTI system similar to a first order system. However, the predominant impact on jitter is not necessarily from $a_{-2}$. The effect of each prior bit is estimated separately from (5), identifying the bit with the largest impact. If $a_{k}$ has the largest impact on DDJ, using the same definition in [4] and replacing $a_{-2}$ by $a_{k}$ we can conclude

$$
D D J_{1}=\Delta t_{k}=\left|\frac{p_{o}\left(t_{0}-k T_{b}\right)}{\left.\frac{d s(t)}{d t}\right|_{t=t_{0}}}\right|,
$$

which is a simple expression that determines the separation of the two impulses in the probability mass function (pmf) of DDJ for a general LTI system. It can be integrated into communication link design or circuit design simulation software to predict the data dependent jitter contribution of the corresponding component in the system.

Equation (9) can be generalized to higher scales of DDJ. For instance, when the prior bit with second largest impact on jitter is also included each pmf in scale one is separated to two pmfs. $\mathrm{DDJ}_{2}$, the scale-two DDJ, is defined as the separation of these two pmfs. From (6) it is easy to show that $\mathrm{DDJ}_{\mathrm{i}}=\Delta t_{i}$, where $\Delta t_{i}$ 
is the sequence of $\Delta t_{k}$ rearranged in decreasing order. $\mathrm{DDJ}_{\mathrm{i}}$ is observable when the system jitter is not dominated by noise (random jitter). In such cases more data transition traces are resolvable from noise in oscilloscope measurements. We will verify equation (9) experimentally in section IV.

\section{EXPERIMENTAL VERIFICATION}

Equation (9) suggests a characterization method for DDJ of any LTI system based only on its step response. The pulse response can be stated in terms of step response as $p(t)=s(t)$ $s\left(t-T_{b}\right)$. Once the step response is characterized (9) can be used to predict DDJ at any data rate. We verify the validity of the results experimentally by comparing the predictions of (9) with measured $\mathrm{DDJ}_{1}$ of several high frequency systems including an integrated CMOS trans-impedance amplifier (TIA). We associate $\mathrm{DDJ}_{1}$ to the separation of the means of two Gaussian distributions, as in Fig. 1, when the jitter histogram at the output of the device under test is measured. For each system, we measure $\mathrm{DDJ}_{1}$ at a bit rate that the system shows significant amount of resolvable data dependent jitter and DDJ is not buried under noise. This reduces sensitivity to measurement errors. The bit rate is always such that data spectrum does not exceed the system bandwidth. This fact demonstrates that while the system bandwidth is large enough to minimize amplitude distortion, DDJ still persists. The jitter histogram is measured after at least 500,000 crossing events are captured by the oscilloscope.

In one set of experiments we carry out the procedure for various off-the-bench systems available in the lab. They include a Mini-Circuits ZFL 1000 -LN driver amplifier with $1 \mathrm{GHz}$ bandwidth, a 9" long $50 \Omega$ copper microstrip on standard FR4 board, a $10.5^{\prime}$ long standard BNC coaxial cable, and an HP $11688 \mathrm{~A}$ microwave lowpass filter with $-3 \mathrm{~dB}$ frequency of $f_{c}=2.8 \mathrm{GHz}$. None of these systems has a simple first order response. Therefore the $\mathrm{DDJ}_{1}$ should be estimated from (9). The measurement results are summarized in TABLE I. Small relative errors in the last column verify the validity of the analytical results for predicting data dependent jitter. For the microstrip line, $a_{-3}$ rather than $a_{-2}$ has the most dominant effect on DDJ and causes the scale-one separation of the threshold crossing times.

TABLE I MEASURED \& ANALYTICAL DDJ 1

\begin{tabular}{|l|c|c|c|c|c|}
\hline \multicolumn{1}{|c|}{ DUT } & Bit Rate & $\begin{array}{c}\text { Measured } \\
D^{2} J_{1}[\mathrm{psec}]\end{array}$ & Bit & $\begin{array}{c}\text { Predicted } \\
\Delta \boldsymbol{t}_{\boldsymbol{k}}[\mathrm{psec}]\end{array}$ & Error \\
\hline \hline ZFL-1000 & $1.3 \mathrm{~Gb} / \mathrm{s}$ & 7.665 & $a_{-2}$ & 7.15 & $6.7 \%$ \\
\hline Microstrip & $10 \mathrm{~Gb} / \mathrm{s}$ & 5.35 & $a_{-3}$ & 5.23 & $2.3 \%$ \\
\hline HP Filter & $1.2 \mathrm{~Gb} / \mathrm{s}$ & 20.5 & $a_{-2}$ & 18.96 & $7.5 \%$ \\
\hline BNC cable & $3 \mathrm{~Gb} / \mathrm{s}$ & 4.6 & $a_{-2}$ & 4.72 & $2.5 \%$ \\
\hline
\end{tabular}

Step response, pulse response and the jitter contributions of four prior bits are plotted in Fig. 3 for two of the systems we tested. $\Delta t_{k}$ is calculated from pulse response using (5). It shows the significance of the pulse response shape of the system and its impact on data dependent jitter at the output. HP 11688A is a filter with $\mathrm{f}_{-3 \mathrm{~dB}}=2.8 \mathrm{GHz}$, while the amplifier $3 \mathrm{~dB}$ bandwidth is $1 \mathrm{GHz}$. Although the filter has much larger bandwidth, it has significantly larger DDJ at similar bit rate $(1.2-1.3 \mathrm{~Gb} / \mathrm{s})$. This can be associated to the pulse response characteristics of the two systems as illustrated in Fig. 3(a) and (b). The pulse response of the filter has larger ringing in its damping tail that dramatically increases the jitter from (5) because the samples of the pulse response at the measurement bit rate $(1.2 \mathrm{~Gb} / \mathrm{s})$ collide with the maxima and minima of the oscillating tail. In fact, bandwidth alone cannot be a complete measure to characterize the DDJ contributions of an LTI system. Although systems with small bandwidth tend to increase DDJ, step response of the system is required to analyze the exact characteristics of output data dependent jitter. Particularly, the system can be designed such that the samples of its pulse response are negligible at integer multiples of bit period to minimize DDJ [6][7], similar to Nyquist's zero-ISI pulse shaping.

To further verify the DDJ estimation theory we also tested the $\mathrm{DDJ}_{1}$ and $\mathrm{DDJ}_{2}$ of an integrated trans-impedance amplifier
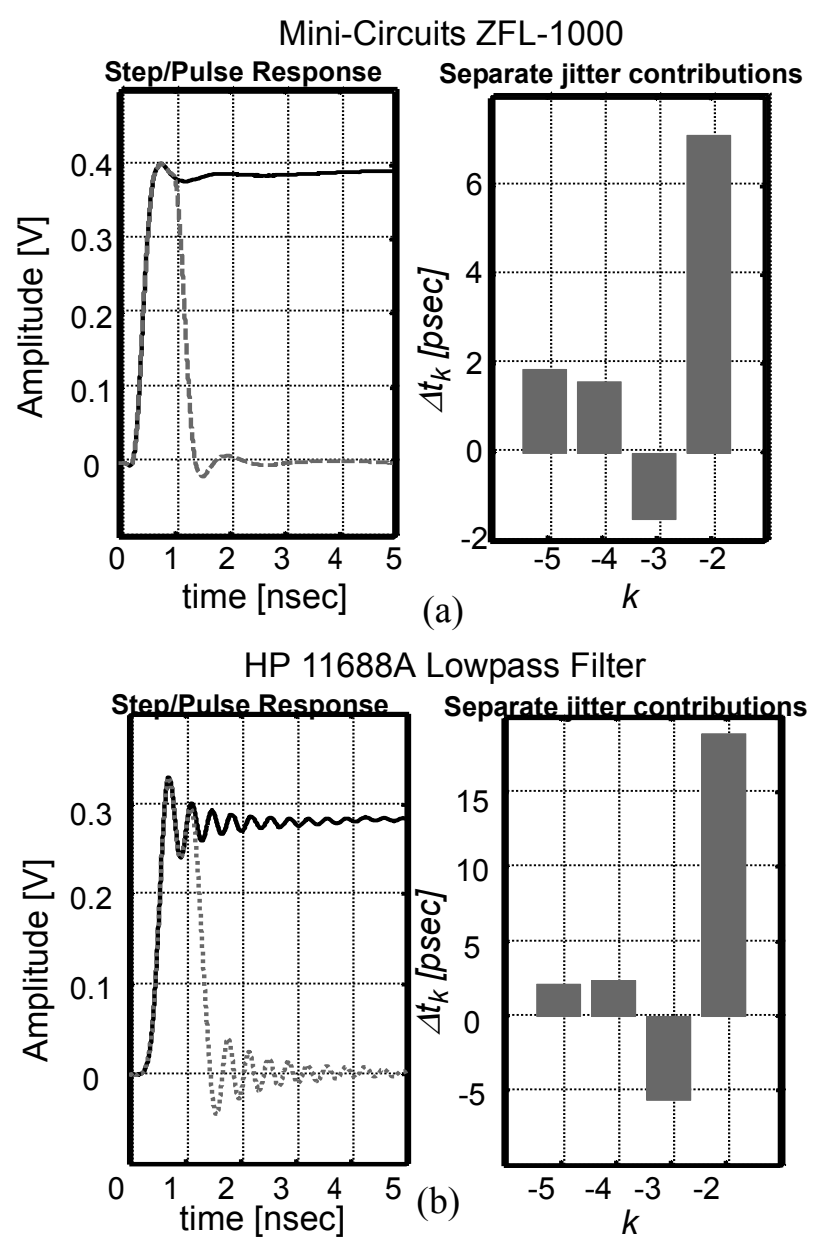

Fig. 3 Step response, pulse response, and jitter contributions of prior bits in (a) Mini-Circuits amplifier (b) HP lowpass filter 


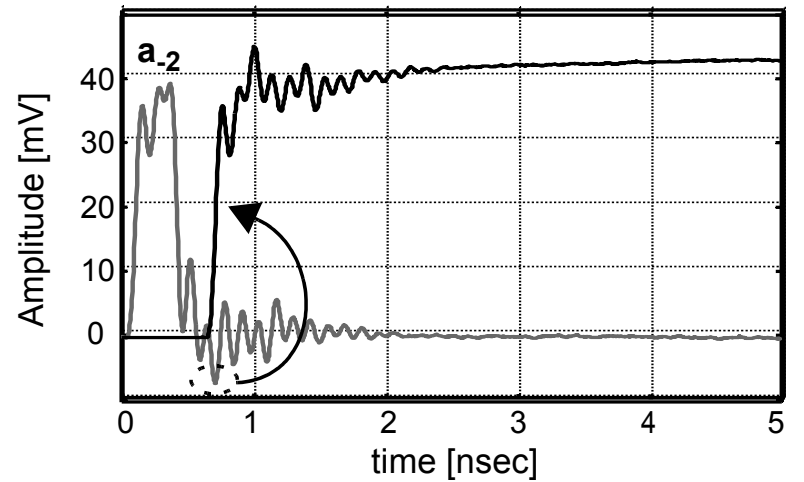

Fig. 4 TIA step response and impact of $a_{-2}$ pulse on $t_{0}$ in a "101" sequence at $3.3 \mathrm{~Gb} / \mathrm{s}$.

(TIA). The TIA was implemented in a $0.18 \mu \mathrm{m}$ BiCMOS technology using only CMOS transistors and demonstrated a 9.2 $\mathrm{GHz} 3 \mathrm{~dB}$ bandwidth [8]. Although the TIA has enough bandwidth to operate at $10 \mathrm{~Gb} / \mathrm{s}$, the reflections from connectors and wirebond mismatches in addition to the amplifier response cause the whole system to have a ringing step response as the measurement shows in Fig. 4. In spite of having enough bandwidth, the TIA along with the measurement setup exhibit large amount of DDJ. We measured DDJ of the TIA at $1.65 \mathrm{~Gb} / \mathrm{s}$ and $3.3 \mathrm{~Gb} / \mathrm{s}$. While the bit rates are within the bandwidth range of the TIA, we observed significant amount of DDJ. The measurement results are summarized in TABLE II. The eye diagram at $1.65 \mathrm{~Gb} / \mathrm{s}$ is shown in Fig. $5(\mathrm{a})$. At $1.65 \mathrm{~Gb} / \mathrm{s}$, DDJ prediction using perturbation method has only $0.85 \%$ error.

TABLE II COMPARING MEASURED $D D J_{1}$ AND PREDICTIONS OF ANALYTICAL EXPRESSION

\begin{tabular}{|c|c|c|c|c|c|}
\hline DUT & Bit Rate & $\begin{array}{c}\text { Measured } \\
\text { DDJ }_{1}[\mathrm{psec}]\end{array}$ & Bit & $\begin{array}{c}\text { Predicted } \\
\Delta t_{k}[\text { psec] }\end{array}$ & Error \\
\hline \hline CMOS TIA & $1.65 \mathrm{~Gb} / \mathrm{s}$ & 6.85 & $a_{-2}$ & 6.8 & $0.85 \%$ \\
\hline CMOS TIA & $3.3 \mathrm{~Gb} / \mathrm{s}$ & 13.6 & $a_{-2}$ & 12.7 & $6.6 \%$ \\
\hline CMOS TIA & $3.37 \mathrm{~Gb} / \mathrm{s}$ & $\mathrm{DDJ}_{2}=5.85$ & $a_{-3}$ & 5.7 & $2.5 \%$ \\
\hline
\end{tabular}

Larger scales of data dependent jitter that are associated with prior bits with less dominant jitter contributions are often smaller than rms of random jitter. Therefore, their impact on total jitter distribution is minimal. However, as discussed in section III the perturbation method can still predict DDJ of larger scales. We measured $\mathrm{DDJ}_{1}$ and $\mathrm{DDJ}_{2}$ of the TIA at 3.37 $\mathrm{Gb} / \mathrm{s}$, where both were observable as Fig. 5(b) illustrates. The measured $\mathrm{DDJ}_{2}$ is compared with calculations in the last row of TABLE II and shows only $2.5 \%$ error.

\section{CONCLUSION}

Data dependent jitter is one type of deterministic jitter that results from residual effects of prior bits on a data threshold crossing time. We proposed a methodology to estimate the DDJ of a general LTI system based on its step response. We

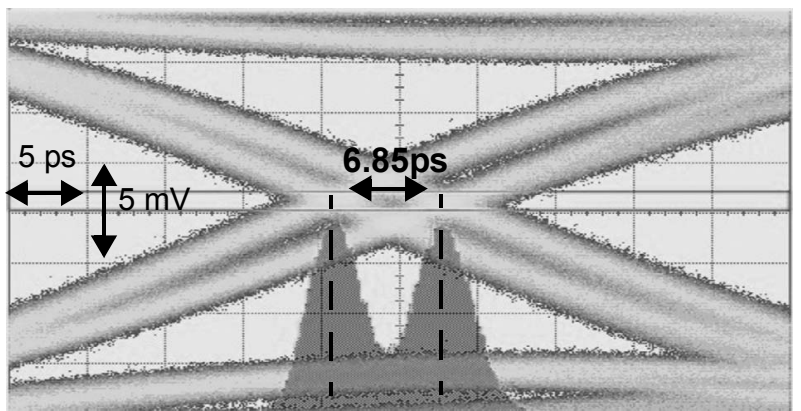

(a)

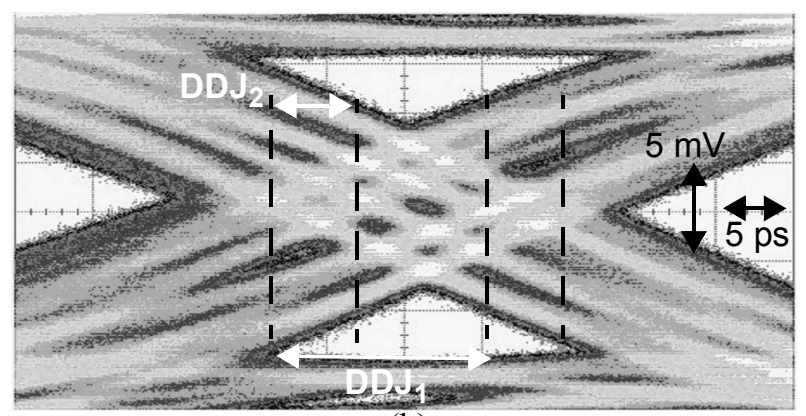

(b)

Fig. 5 TIA eye diagram when $\mathrm{DDJ}_{1}$ and $\mathrm{DDJ}_{2}$ are observable (a) $1.65 \mathrm{~Gb} / \mathrm{s}$ (b) $3.37 \mathrm{~Gb} / \mathrm{s}$.

highlighted that $3 \mathrm{~dB}$ bandwidth does not characterize DDJ of the system completely and the shape of the step response is important. Finally, we verified the validity of the analytical results experimentally for several gigahertz range systems.

\section{REFERENCES}

[1] IEEE 802.3 working group web page: http://www.ieee802.org/3.

[2] International Committee for Information Technology Standardization (INCITS), Fibre Channel - Methodologies for Jitter and Signal Quality Specification- Tech. Rep. REV 10.0, March 2003.

[3] M. Shimanouchi, "An Approach to Consistent Jitter Modeling for Various Aspects and Measurement Methods," Proc. of IEEE Int. Test Conf., pp. 848-857, Oct.-Nov. 2001.

[4] J. Buckwalter, B. Analui, and A. Hajimiri, "Predicting DataDependent Jitter," IEEE Trans. Circs. \& Syst. II: Technical Briefs, vol. 51, no. 9, pp. 453-457, September 2004.

[5] ---, "Data-Dependent Jitter and Crosstalk-Induced Bounded Uncorrelated Jitter in Copper Interconnects," IEEE MTT-S Int. Microwave Symp. Dig., vol. 3, pp. 1627-1630, June 2004.

[6] R. A. Gibby and J. W. Smith, "Some extensions of Nyquist's telegraph transmission theory," The Bell System Tech. J., vol. 44, pp. 1487-1510, Sept. 1965.

[7] J. C. Y. Huang, K. Feher, and M. Gendron, "Techniques to Generate ISI and Jitter-Free Bandlimited Nyquist Signals and a Method to Analyze Jitter Effects," IEEE Trans. Comm., vol. 27, no. 11, pp. 1700-1711, Nov. 1979.

[8] B. Analui and A. Hajimiri, "Bandwidth Enhancement for Transimpedance Amplifiers," IEEE J. Solid-State Circs, vol. 39, no.8, pp. 1263-1270, Aug. 2004. 